\title{
The Role of Prophylactic Antibiotic Use in Prevention of Endophthalmitis Following Intravitreal Injection of Anti-Vascular Endothelial Growth Factor Agents: A Meta-Analysis
}

\author{
Sonia B. Dhoot, Nancy Kunjukunju, Nelson Sabates \\ Department of Ophthalmology, University of Missouri, Kansas City, USA. \\ Email: brars522@gmail.com,nkunjukunju@gmail.com, sabatesn@sabateseye.com
}

Received February $22^{\text {nd }}, 2013$; revised March $28^{\text {th }}, 2013$; accepted May $1^{\text {st }}, 2013$

Copyright (C) 2013 Sonia B. Dhoot et al. This is an open access article distributed under the Creative Commons Attribution License, which permits unrestricted use, distribution, and reproduction in any medium, provided the original work is properly cited.

\begin{abstract}
Purpose: To determine the role of prophylactic antibiotic use in preventing endophthalmitis following intravitreal injections of Anti-Vascular Endothelial Growth Factor Agents (anti-VEGF). Methods: A meta-analysis was conducted for studies reporting the rates of endophthalmitis following injection of anti-vascular endothelial growth factor agents for a variety of disorders. Inclusion criteria included reporting how many patients were given post-injection antibiotic drops, and how many patients were diagnosed with endophthalmitis (both with and without antibiotic drops). Results: The reported rate of endophthalmitis following intravitreal anti-VEGF agents has varied significantly based on the study, with a range between $0.009 \%$ to $0.87 \%$. In our meta-analysis, the rate of endophthalmitis in patients receiving postinjection antibiotics was found to be $0.081 \%$, compared to $0.072 \%$ of patients who did not receive antibiotics. This difference was not found to be statistically significant. Conclusion: Although the rate of endophthalmitis is extremely low following intravitreal anti-VEGF injections, the controversy regarding the best prevention of this dreaded complication continues. According to our meta-analysis, the answer does not seem to lie in post-injection antibiotic use.
\end{abstract}

Keywords: Endophthalmitis; Intravitreal Injections; Anti-VEGF

\section{Introduction}

With the increasing use of intravitreal anti-VEGF agents (pegaptanib, bevacizumab, and ranibizumab) for multiple retinal conditions, including exudative age related macular degeneration, clinically significant macular edema, and proliferative diabetic retinopathy, a new subset of endophthalmitis has emerged. This rate varies based on the study, but ranges from $0.009 \%$ to $0.87 \%$, and will continue to rise with the increasing use of these injections [1-6]. Although the incidence is low, endophthalmitis is a devastating complication, which may cause apoptosis of ganglion cells, bipolar cells and photoreceptors, lead to retinal detachments, and potentially cause severe and permanent loss of vision [2,7]. Patients who have retinal detachments secondary to endophthalmitis are less likely to regain their baseline vision even with treatment [2].

Shah et al. reviewed possible risk factors for the development of endophthalmitis, including lid speculum use, conjunctival displacement during injection, the hemi- sphere of injection, and bevacizumab vs ranibizumab, and found no statistically significant difference between any of the above groups. The only statistically significant variable they found to decrease the rate of endophthalmitis was the use of topical povidone-iodine to sterilize the ocular surface [2]. Both this study and the study by Mezad-Koursh et al. did not examine the use of postinjection antibiotics as an independent variable; all patients in both groups routinely received antibiotics postinjection [2-3]. It has been shown that the use of povidone-iodine on the ocular surface is just as effective as a combination of preinjection antibiotic drops along with povidone-iodine, and most ophthalmologists therefore do not use any preinjection antibiotics [8].

Of the few cases of post-injection endophthalmitis reported in the literature, there is a significant percentage that had negative cultures $(33 \%-70 \%)[1-3,6]$. Of the cases in which cultures were positive, the most common organisms were coagulase-negative Staphylococcus spe- 
cies, Streptococcus species, followed by Bacillus cereus, and Enterococcus faecalis [1,2]. Injection-related endophthalmitis is believed to be secondary to local contamination. Streptococcus is responsible for more endophthalmitis cases following intravitreal injections than in postsurgical endophthalmitis cases $[9,10]$. Because Streptococcus is also found in normal salivary flora, its increased rate in post-injection endophthalmitis cases has been postulated to be secondary to droplet spread of the organism [11-14]. Therefore, although the current recommendations do not include the use of a mask while performing intravitreal injections, their use may be helpful in reducing the number of Streptococcus-related endophthalmitis cases [1].

Multiple recent studies have failed to show any statistically significant difference in the use of postinjection antibiotics and the rate of endophthalmitis [4-6]. However, despite these studies, more than $2 / 3$ of retinal specialists still use topical antibiotics after injections according to a recent survey $[2,3,15]$. When prescribed, the antibiotic of choice varies based on clinician, with a fluoroquinolone being by far the most common class used. However, many studies have shown that different generations of fluoroquinolones have differing minimum inhibitory concentrations for bacterial organisms in the vitreous [16-18]. This meta-analysis was performed to survey the literature and help further delineate the role of post-injection antibiotics in the prevention of end ophthalmitis.

\section{Materials and Methods}

A literature search was conducted using the National Library of Medicine PubMed (www.pubmed.gov). All articles containing the keywords "endophthalmitis AND anti-vascular endothelial growth factor" and "antibiotics AND anti-vascular endothelial growth factor" were reviewed (most recent search: February 2, 2013). The references of these papers were also subsequently reviewed. A meta-analysis was performed using studies with the following criteria: the use of post-injection antibiotics as an independent variable in determining the rate of endophthalmitis and analysis of only cases in which intravitreal anti-VEGF agents were administered. Cases in which antibiotics were studied for endophthalmitis prophylaxis in surgical cases or following intravitreal triamcinolone or other steroids were excluded. In some studies, the decision of whether or not to prescribe post-injection antibiotics was left up to the discretion of the ophthalmologist performing the injection, and specific numbers of patients with and without antibiotics were not included. These studies were excluded from the meta-analysis. When using antibiotic drops, all studies in the meta-analysis used a fourth-generation fluoroquinolone. A large table was made compiling all the patients who were diagnosed with endophthalmitis and whether or not they were given post-injection antibiotics. This data was then analyzed using a chi-square test for statistical significance.

\section{Results}

The initial PubMed search returned a total of 16 articles. After the appropriate inclusion criteria were applied and the additional reference articles were included, a total of seven articles remained for analysis. The relevant information from these articles is summarized in Table 1.

Four of the seven studies routinely used post-injection antibiotics $[2,3,19,20]$ while the remaining three studies had two groups-one which received post-injection antibiotics and one which did not [4-6].

A total of 72,823 injections were considered in the meta-analysis, 61,744 of which received post-injection antibiotics and 11,079 of which did not. The rate of endophthalmitis in the antibiotic group was found to be $0.081 \%$, whereas the rate of endophthalmitis in the control group was $0.072 \%$. This difference was not found to be statistically significant $(\mathrm{P}>0.50)$.

\section{Discussion}

The meta-analysis of the rates of endophthalmitis in patients who received post-injection antibiotics compared to those patients without antibiotics showed a slightly increased risk of endophthalmitis in patients who used antibiotics. This rate however was not found to be statistically significant, and may have been secondary to the fact that there were many fewer studies in which no antibiotics were used.

The decision between administering antibiotic drops for a few days following injections versus the threat of possible endophthalmitis seems to be an obvious one at first glance. The antibiotic of choice when used varies based on clinician, and as many prior studies have shown, many of these topical antibiotics may not even reach sufficient inhibitory concentrations to kill organisms present in the vitreous [16,19-20]. It is therefore no surprise that endophthalmitis cannot be prevented with topical antibiotic use in these cases once the organisms have already infiltrated the vitreous.

The use of povidone-iodine on the conjunctiva prior to the injection remains to be the only proven modifiable risk factor in preventing this dreaded complication $[1,4$, 5]. Streoptococcus has been found to be the culprit in more post-injection endophthalmitis cases than it has in postsurgical endophthalmitis $[9,10]$. This has led to a rising concern for droplet transmission of Streptococcus species from oral flora during injections, and many oph- 
Table 1. Studies reporting rates of endophthalmitis that were included in the meta-analysis.

\begin{tabular}{|c|c|c|c|c|c|c|c|}
\hline \multirow[b]{2}{*}{ Study } & \multicolumn{3}{|c|}{+ Antibiotics } & \multicolumn{2}{|c|}{-Antibiotics } & \multirow[b]{2}{*}{$\begin{array}{l}\text { Rate } \\
(\%)\end{array}$} & \multirow[b]{2}{*}{ Total \# of injection } \\
\hline & +Endophthalmitis & -Endophthalmitis & $\begin{array}{l}\text { Rate } \\
(\%)\end{array}$ & +Endophthalmitis & -Endophthalmitis & & \\
\hline $\begin{array}{l}\text { Pilli et al. } \\
\text { [19] }\end{array}$ & 3 & 10,251 & 0.029 & N/A & N/A & N/A & 10,254 \\
\hline $\begin{array}{l}\text { Lima et al. } \\
\quad[20]\end{array}$ & 2 & 3066 & 0.065 & N/A & N/A & N/A & 3068 \\
\hline $\begin{array}{l}\text { Shah et al. } \\
\text { [2] }\end{array}$ & 23 & 27,713 & 0.083 & N/A & N/A & N/A & 27,736 \\
\hline $\begin{array}{l}\text { Mezad-Koursh } \\
\quad \text { et al. [3] }\end{array}$ & 4 & 3072 & 0.130 & N/A & N/A & N/A & 3076 \\
\hline Bhatt et al. [4] & 5 & 2282 & 0.219 & 5 & 2475 & 0.202 & 4767 \\
\hline $\begin{array}{c}\text { Bhavsar et al. } \\
\text { [5] }\end{array}$ & 6 & 4688 & 0.128 & 1 & 3332 & 0.030 & 8027 \\
\hline $\begin{array}{l}\text { Cheung } \\
\text { et al. [6] }\end{array}$ & 7 & 10,622 & 0.066 & 2 & 5264 & 0.038 & 15895 \\
\hline TOTAL & 50 & 61,694 & 0.081 & 8 & 11,071 & 0.072 & 72,823 \\
\hline
\end{tabular}

thalmologists have therefore started using masks while performing injections. Further research needs to be done to study the relationship between the use of masks and the rate of endophthalmitis.

The specific antibiotic and dosing regimen varied in some studies, and were left up to the discretion of the ophthalmologist administering the injection. This varied regimen could be a potential source of bias in the above meta-analysis. Because antibiotic prophylaxis was not directly being studied in any of the above studies, the decision of whether or not to use antibiotics was also left up to the discretion of the clinician. The majority of clinicians opted to use post-injection antibiotics, which resulted in a greater number of total cases in the antibiotic group. In an ideal situation, the meta-analysis would have contained an equal number of total cases in the antibiotic and control groups which would have allowed for a more balanced comparison.

\section{Conclusion}

Despite many recent studies claiming the only significant modifiable risk factor in preventing endophthalmitis is the use of povidone-iodine [1-2,4], the use of post-injection antibiotics remains commonplace in many ophthalmology practices. Our meta-analysis is in agreement with some recent studies that suggest that there is no role for post-injection antibiotics following anti-vascular endothelial growth factor injections [4,5]. Implementing a guideline to limit antibiotic prophylaxis after intravitreal injections could help reduce costs in the face of a dramatically increasing rate of injections per year.

\section{REFERENCES}

[1] C. McCannel, "Meta-Analysis of Endophthalmitis after Intravitreal Injection of Anti-Vascular Endothelial Growth Factor Agents," Retina, Vol. 31, No. 4, 2011, pp. 654661.

[2] C. P. Shah, S. J. Garg, J. F. Vander, et al., "Outcomes and Risk Factors Associated with Endothelial Growth Factor Agents," Ophthalmology, Vol. 118, No. 10, 2011, pp. 2028-2034. doi:10.1016/j.ophtha.2011.02.034

[3] D. Mezad-Koursh, M. Goldstein, G. Heilwail, et al., "Clinical Characteristics of Endophthalmitis after an Injection of Intravitreal Antivascular Endothelial Growth Factor," Retina, Vol. 30, No. 7, 2010, pp. 1051-1057. doi:10.1097/IAE.0b013e3181cd47ed

[4] S. S. Bhatt, K. E. Stepien and K. Joshi, "Prophylactic Antibiotic Use after Intravitreal Injection: Effect on Endophthalmitis Rate," Retina, Vol. 31, No. 10, 2011, pp. 2032-2036. doi:10.1097/IAE.0b013e31820f4b4f

[5] A. R. Bhavsar, C. R. Stockdale, F. L. Ferris, et al., "Update on Risk of Endophthalmitis after Intravitreal Drug Injections and Potential Impact of Elimination of Topical Antibiotics," Archives of Ophthalmology, Vol. 130, No. 6, 2012, p. 227.

[6] C. S. Cheung, A. W. Wong, A. Lui, et al., "Incidence of Endophthalmitis and Use of Antibiotic Prophylaxis after Intravitreal Injections," Ophthalmology, Vol. 119, No. 8, 2012, pp. 1609-1614. doi:10.1016/j.ophtha.2012.02.014

[7] N. M. Pharmakakis, I. K. Petropoulos, C. D. Georgakou- 
poulos, et al., "Apoptotic Mechanisms within the Retina in Staphylococcus epidermidis Experimental Endophthalmitis," Graefes Archives for Clinical and Experimental Ophthalmology, Vol. 247, 2009, pp. 667-674. doi:10.1007/s00417-008-0996-Z

[8] J. M. Moss, S. R. Sanslo and C. N. Ta, "A Prospective Randomized Evaluation of Topical Gatifloxacin on Conjunctival Flora in Patients Undergoing Intravitreal Injections," Ophthalmology, Vol. 116, No. 8, 2009, pp. 14981501. doi:10.1016/i.ophtha.2009.02.024

[9] The Endophthalmitis Vitrectomy Study Group, "Microbiologic Factors and Visual Outcome in the Endophthalmitis Vitrectomy Study," American Journal of Ophthalmology, Vol. 122, No. 6, 1996, pp. 830-846.

[10] Endophthalmitis Vitrectomy Study Group, "Results of the Endophthalmitis Vitrectomy Study. A Randomized Trial of Immediate Vitrectomy and of Intravenous Antibiotics for the Treatment of Postoperative Bacterial Endophthalmitis," Archives of Ophthalmology, Vol. 113, No. 12, 1995, pp. 1479-1496. doi:10.1001/archopht.1995.01100120009001

[11] E. Veringa, A. VanBelkum and H. Schellekens, "Iatrogenic Meningitis by Streptococcus salivarius Following Lumbar Puncture," Journal of Hospital Infection, Vol. 29, No. 4, 1995, pp. 316-318. doi:10.1016/0195-6701(95)90283-X

[12] S. W. O'Kelly and D. Marsh, "Face Masks and Spinal Anesthesia," British Journal of Anaesthesia, Vol. 710, 1993, p. 239. doi:10.1093/bja/70.2.239

[13] H. A. McLure, C. A. Talboys, S. M. Yentis, et al., "Surgical Face Masks and Downward Dispersal of Bacteria," Anesthesia, Vol. 53, 1998, pp. 624-626. doi:10.1046/j.1365-2044.1998.435-az0528.x

[14] M. Trautmann, P. M. Lepper and F. J. Schmitz, "Three
Cases of Bacterial Meningitis after Spinal and Epidural Anesthesia," European Journal of Clinical Microbiology and Infectious Diseases, Vol. 21, No. 1, 2002, pp. 43-45. doi:10.1007/s10096-001-0643-7

[15] A. E. Green-Simms, N. S. Ekdawi and S. J. Bakri, "Survey of Intravitreal Injection Techniques Among Retinal Specialists in the United States," American Journal of Ophthalmology, Vol. 151, No. 2, 2011, pp. 329-332. doi:10.1016/j.ajo.2010.08.039

[16] S. M. Hariprasad, K. J. Blinder, G. K. Shah, et al., "Penetration Pharmacokinetics of Topically Administered 0.5\% Moxifloaxcin Ophthalmic Solution in Human Aqueous and Vitreous," Archives of Ophthalmology, Vol. 123, No. 1, 2005, pp. 39-44. doi:10.1001/archopht.123.1.39

[17] O. Cekic, C. Batman, U. Yasar, et al., "Penetration of Ofloxacin in Human Aqueous and Vitreous Humors Following Oral and Topical Administration," Retina, Vol. 18, No. 6, 1998, pp. 521-525. doi:10.1097/00006982-199806000-00005

[18] O. Cekic, C. Batman, U. Yasar, et al., "Human Aqueous and Vitreous Humor Levels of Ciprofloxacin Following Oral and Topical Administration," Eye, Vol. 13, No. 4, 1999, pp. 555-558. doi:10.1038/eye.1999.137

[19] S. Pilli, A. Kotsolis, R. F. Spaide, et al., "Endopthalmitis Associated with Intravitreal Anti-Vascular Endothelial Growth Factor Therapy Injections in an Office Setting," American Journal of Ophthalmology, Vol. 29, 2008, pp. 153-154.

[20] L. H. Lima, S. A. Zweifel, M. Engelbert, et al., "Evaluation of Safety for Bilateral Same-Day Intravitreal Injections of Antivascular Endothelial Growth Factor Therapy," Retina, Vol. 29, No. 9, 2009, pp. 1213-1217. doi:10.1097/IAE.0b013e3181b32d27 